\title{
Seguridad corporativa y cultura de seguridad en estudiantes de ingeniería - Universidad Nacional de Moquegua
}

\author{
Corporate security and safety culture in engineering students - \\ Universidad Nacional de Moquegua
}

Víctor Adrián Ponce Estrada ${ }^{1}$

Recibido: 14/09/2020 - Aprobado: 05/04/2021 - Publicado: 18/06/2021

\begin{abstract}
RESUMEN
Esta investigación tiene como objetivo determinar la relación de la seguridad corporativa y la cultura de la seguridad minera en los estudiantes de Ingeniería de Minas de la Universidad Nacional de Moquegua, 2019; por su finalidad es de tipo básica, por su profundidad es correlacional. De diseño no experimental, transeccional (o transversal), descriptiva-correlacional; la correlación de la variables se determinó con el Rho de Spearman y la aceptación rechazó de la hipótesis se estableció mediante la significatividad bilateral. La muestra fue 68 estudiantes de Ingeniería Minera de la Universidad Nacional de Moquegua, a quienes se aplicó una encuesta tipo Likert, constituida por 40 ítems. Los resultados indican un Rho de Spearman de 0.659 y un nivel de significatividad de $p=0.0015704109<\alpha=0.01$. Concluyendo que existe relación positiva y significativa entre la seguridad corporativa y la cultura de la seguridad minera en los estudiantes de Ingeniería de Minas de la UNAM.
\end{abstract}

Palabras clave: Seguridad corporativa; cultura de seguridad; seguridad minera.

\begin{abstract}
This research aims to determine the relationship of corporate security and the culture of mining safety in students of Mining Engineering at the National University of Moquegua, 2019; by its purpose it is of basic type, by its depth it is correlational. Non-experimental, transectional (or transversal), descriptive-correlational design; the correlation of the variables was determined with Spearman's Rho and the acceptance rejected of the hypothesis was established by means of the bilateral significance. The sample was 68 students of Mining Engineering from the National University of Moquegua, to whom a Likert-type survey was applied, consisting of 40 items. The results indicate a Spearman's Rho of 0.659 and a level of significance of $p=$ $0.0015704109<\alpha=0.01$. Concluding what there is a positive and significant relationship between corporate security and the culture of mining safety in students of Mining Engineering at UNAM.
\end{abstract}

Keywords: Corporate security; safety culture; mining security.

1 Ingeniero de Minas. Docente Ordinario en la Escuela de Ingeniería de Minas de la Universidad Nacional de Moquegua; Ingeniero de Seguridad en Jamal Contratistas Generales E.I.R.L. E-mail: victoradrian 2@hotmail.com - ORCID: https://orcid.org/0000-0002-7156-1898 


\section{INTRODUCCIÓN}

La minería, es una de las actividades extractiva vinculada al desarrollo económico de las sociedades humanas en el ámbito regional y nacional (Herrera \& Parra, 2018), que en el caso de Perú, es una actividad presente desde su época preinca e inca, aunque a nivel económico tenía un nivel secundario, pero en la época colonial se constituye en una actividad económica principal (IPE, 2017). Actualmente -señala Valdez (2016)- la minería en Perú es una de las actividades económicas relevantes con una de las mayores reservas mundiales de plata, cobre, zinc, plomo, estaño y oro.

En la explotación minera se tiene la minería subterránea y la minería a cielo abierto, que difieren en su operatividad, condiciones de trabajo, riesgos, exposición y consecuencias (Cárdenas et al., 2017), por ende, el riesgo es inherente a esta actividad económica, estando presente la exposición a los accidentes -que son el efecto de actos erróneos que realizan los trabajadores, o porque los equipos, herramientas, maquinarias o lugares de trabajo no se encuentran en condiciones apropiadas (Seguel et al., 2017); en la minería, los sucesos laborales con daño humano y la accidentalidad son acaecimientos presentes en esta actividad.

Los accidentes de trabajo, según el Reglamento de Seguridad y Salud Ocupacional en Minería (Art. $7^{\circ}$ ), son todas las ocurrencias repentinas que se dan por acción laboral y generen en el trabajador lesiones orgánicas, perturbación funcional, invalidez o muerte (Diario El Peruano, 2016) Ministerio de Energía y Minas; de manera que los accidentes de trabajo son eventos fortuitos laborales, asociados a factores como las condiciones de seguridad laboral que devienen en efectos de índole personal, familiar y social (Mejia et al., 2015), por lo que es menester que las empresas mineras tomen una política comprometida corporativamente con la seguridad laboral.

La seguridad corporativa, es cuando la seguridad se da desde la empresa misma, partiendo desde lo que franquea la ley de seguridad laboral, donde la estrategia y el director de seguridad tienen el rol relevante (Vázquez, 2018), una definición básica, de la cual Ceja (2019) recoge el aspecto estratégico, al precisar que la seguridad corporativa constituye una visión global y estratégica que tiene como objetivo identificar y mitigar los riesgos y vulnerabilidades que confluyen en la organización, administrarlos con efectividad holística y determinar la capacidad resiliente de la empresa, debiéndose garantizar el apoyo de la dirección que posibilite la creación de conciencia y disciplina de la seguridad organizacional; y decantando en la política de seguridad corporativa, que como aprecia Iberdrola (2013), consiste en establecer los principios básicos de acción que garanticen la eficaz protección de las personas, de los activos físicos y logísticos, así como establecer la connotación resiliente de la organización, involucrando a los cuatros activos básicos de la empresa, los recursos humanos, financieros, administrativos y operativos. Concretamente, Muñoz (2016) postula la seguridad corporativa como la función que de manera preventiva identifica, gestiona y atenúa eficientemente las amenazas a la seguridad y la resiliencia de la organización, valiéndose de la concurrencia de las políticas, acciones, recursos humanos, recursos técnicos y organización amalgamados para proteger a las personas y los activos de la empresa, basados en una estrategia transversal en toda la organización; derivándose en que la función de seguridad corporativa es una acción transversal en la estrategia de las empresas.

Un aspecto relevante de la estrategia en la seguridad corporativa es trabajar en el convencimiento y la creación de conciencia (Ceja, 2019), implicando el desarrollo de la actitud de los trabajadores para con la seguridad.

La cultura de la seguridad parte del concepto de cultura. Iglesias et al (2016) anotan que cultura es un estilo de vida de una agrupación de individuos, también es un esquema de símbolos conocidos universalmente; pero pondera a la cultura como las particularidades del conjunto de personas, y agregan que constituye la concurrencia del desarrollo intelectual, mental y estético, constituida por tres elementos: las ideas, los valores y las emociones (Iglesias et al, 2016); yuxtaponiendo la cultura objetiva, que es un medio para que la persona o grupo de personas logren asimilar la cultura de un grupo social (como el de una organización), tal como es el caso de las normas de seguridad -vistas como un valor- orientan la conducta de las personas hacia las buenas prácticas de seguridad. En suma, en opinión de Iglesias et al (2016) la cultura es el multiverso simbólico, integrado por ideas, valores y emociones interiorizadas en la conciencia (comprensión, conocimiento, percepción) y la comunicación contextualizadas con la cotidianeidad (como la laboral) de un grupo social para lograr ser mejores en la medida que se experimenten, construyan significados y orientaciones vivenciales.

Con ello, se tiene que la cultura de la seguridad es un patrón constituido por los comportamientos personales y organizacionales instituidos en valores, reglas, normas, creencias, costumbres que compartes los miembros de una organización a fin de mitigar los daños al que están expuestos (Committee of Experts on Management of Safety and Quality in Health Care SP-SQS, 2005), definición que recoge los aspectos básicos de la cultura, que ICSI (2017) la mejora al indicar que la cultura de seguridad es el acervo compartido de las formas de proceder, de pensar entre los miembros de la organización referente al proceso de afrontar los riesgos inherentes a las actividades de la empresa.

Los riesgos laborales en parte pueden ser atenuados con la aplicación de la Seguridad Basada en el Comportamiento (SBC), que es una metodología para observar el comportamiento de las personas en su medio laboral a fin de coadyuvar a mejorar los comportamientos seguros, controlar los comportamientos de riesgo e interiorizar una cultura de seguridad en los trabajadores (Cajia \& Silva, 2019).

Por su parte, Pérez (2015) indica que en 1980 surgió la concepción de cultura de seguridad, desde la perspectiva de cultura preventiva e insertada en el Modelo OHSAS 18001 (Occupational Health and Safety Assessment Series) de 1999, basada en especificar el accionar de los Sistemas de gestión de la Seguridad y Salud en el Trabajo; también se tiene el sistema International Safety Training and Technology Company (ISTEC) que establece estándares de excelencia en seis áreas de riesgo de la 
empresa, tales como en el área de organización y control, el área de seguridad ocupacional y protección física, así como en higiene y medicina de la salud laboral, en el área de seguridad en procesos, en la zona de prevención y protección contra incendios, y en el área de protección ambiental; a ellos, se suma el modelo de Parker dirigido a crear una cultura preventiva, es un ascenso progresivo de la ambiente cultural de la seguridad en la empresa, categorías que va de lo patológico, siguiendo a lo reactivo, formalista, proactivo y finalizando en lo generativo; se cuenta con el modelo DuPont, denominada STOP, que es un proceso observacional basado en las acciones de decidir, detener, observar, actuar, reportar; otro método, el de las 5S: clasificación, orden, limpieza, estandarización y mantenimiento de la disciplina; y, el modelo DNV (Det Norske Veritas - DNV). Modelos que aportan a la seguridad corporativa y la cultura de la seguridad minera.

Cada modelo de la cultura de seguridad reporta componentes básicos, como precisa Vítolo (2016) son: (i) reconocer que en la empresa existen actividades de alto riesgo; (ii) compartir entre el personal la insostenibilidad de la gravedad de los daños que pueden ocasionar las actividades de alto riesgo; (iii) tener un ambiente correctivo de las actividades de riesgo; (iv) reconocimiento a la colaboración interna; (v) trabajo en equipo; (vi) valorar la prioridad de la integridad física los trabajadores; (vii) motivar el compromiso de los líderes de la empresa con la seguridad; y, (viii) valorar el compromiso del personal con la seguridad.
Establecer la cultura de seguridad efectiva en una empresa pasa por conocer el estado, nivel, punto del proceso de seguridad se encuentra, de manera que a partir de ello se planifique estratégicamente las acciones para avanzar en este proceso, que coadyuva a aplicar la denominada Curva de Bradley.

La curva de Bradley, apunta Oliva (2016) es una herramienta de gestión de la seguridad corporativa que tienen las empresas para conocer en qué punto se encuentra la seguridad organizacional en el camino para lograr la cultura de seguridad efectiva; establecido el punto de madurez situacional de la cultura de seguridad y como punto de quiebre se debe efectuar actividades destinadas a lograr mejores peldaños de seguridad; esta curva tiene tres ejes básicos, como el liderazgo, la organización y los procesos y actividades (Oliva, 2016). En la Figura 1, la curva de Bradley está divida en cuatro segmentos: fase reactiva, fase dependiente, fase independiente y fase interdependiente (Oliva, 2016).

En la fase reactiva, la dirección, mandos y trabajadores de la empresa son indiferentes a las acciones preventivas de seguridad, por considerar que los accidentes son eventualidades desafortunadas, ante lo cual el trabajador debe actuar por institnto, luego se debe actuar con medidas correctivas improvisadas sin tener encuenta el meollo del problema, de manera que no existe participación, responsabilidad ni compromiso por la seguridad de ninguno de los agentes activos de la empresa.

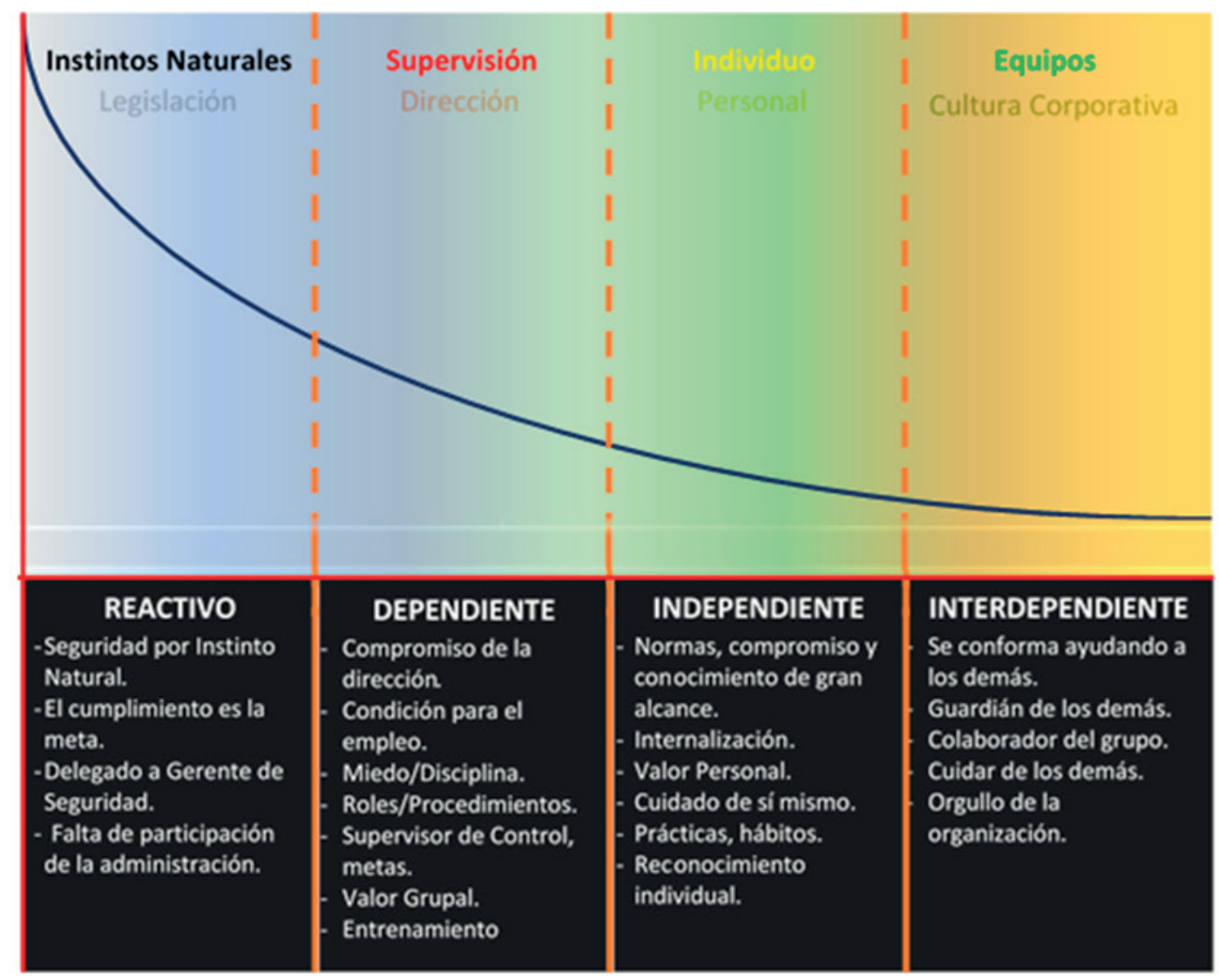

Figura 1. Curva de Bradley. Fuente: Oliva (2016) 
En la fase dependiente, la dirección está comprometida con la seguridad, pero la salvaguardia es responsabilidad de quienes están a cargo de ella, que mediante la supervisión del cumplimiento de reglas, normas y procedimientos de seguridad la accidentabilidad se reduce, caso contrario se debe a su incumplimiento por parte del trabajador, por ende, en seguridad el trabajador no es parte de la solución. Oliva (2016) indica que según la Curva de Bradley la actividad minera se encuentra en la fase dependiente (Figura 2), posicionamiento encaminado a mejorar en la mdida que se mejore los aspectos legales y se tome conciencia del rol de la seguridad.

En la fase independiente, la seguridad involucra a la dirección, a los encargados de la seguridad y a los trabajadores de la empresa, a todos se inculca el compromiso, la responsabilidad, el cumplimiento de la normatividad, de los procedimientos, asi como el ejercicio formativo de la seguridad, donde cada uno es responsable de su seguridad; de manera que en esta fase se inculca el alto sentido de la autoprotección y con ello los trabajadores trabajan para ser parte de la solución de la seguridad de la empresa.

En la fase independiente no es admisible que alguien se lesione por efecto del trabajo y se asume cabalmente el compromiso de cero accidentes; para ello, la seguridad se basa en el trabajo en equipo, donde la dirección de la empresa, los encargados de la seguridad y los trabajadores están involucrados colectivamente en la seguridad, implicando a la comunicación, capacitación, cooperación e integración como elementos claves para el logro de las metas y objetivos de la seguridad.

\section{MÉTODOS}

Esta investigación es de nivel descriptivo, transversal, por su profundidad es de nivel correlacional (Hernández \& Mendoza, 2018), en la medida que provee da data necesaria de la influencia de una variable (seguridad corporativa) sobre otra variable (cultura de la seguridad minera) (Namakforoosh, 2015), es de diseño transeccionalcorrelacional; con una muestra censal de 68 estudiantes de la Facultad de Ingeniería Minera de la Universidad Nacional de Moquegua. Para el trabajo de campo se aplicó la técnica de la encuesta, para lo cual tres expertos validaron los instrumentos de investigación de recolección de datos: (1) Encuesta de percepción de la seguridad y (2) Cuestionario cultura de la seguridad minera aplicados a los estudiantes de Ingeniería Minera de los ciclos VII, VIII, IX y X, la data fue procesada con el programa SPSS v. 25 y Excel v. 14.0, que dieron estadísticos descriptivos e inferenciales; donde las relación de las variables es determinada por el coeficiente Rho de Spearman y la aceptación o rechazo de la hipótesis se realizó con la significatividad bilateral.

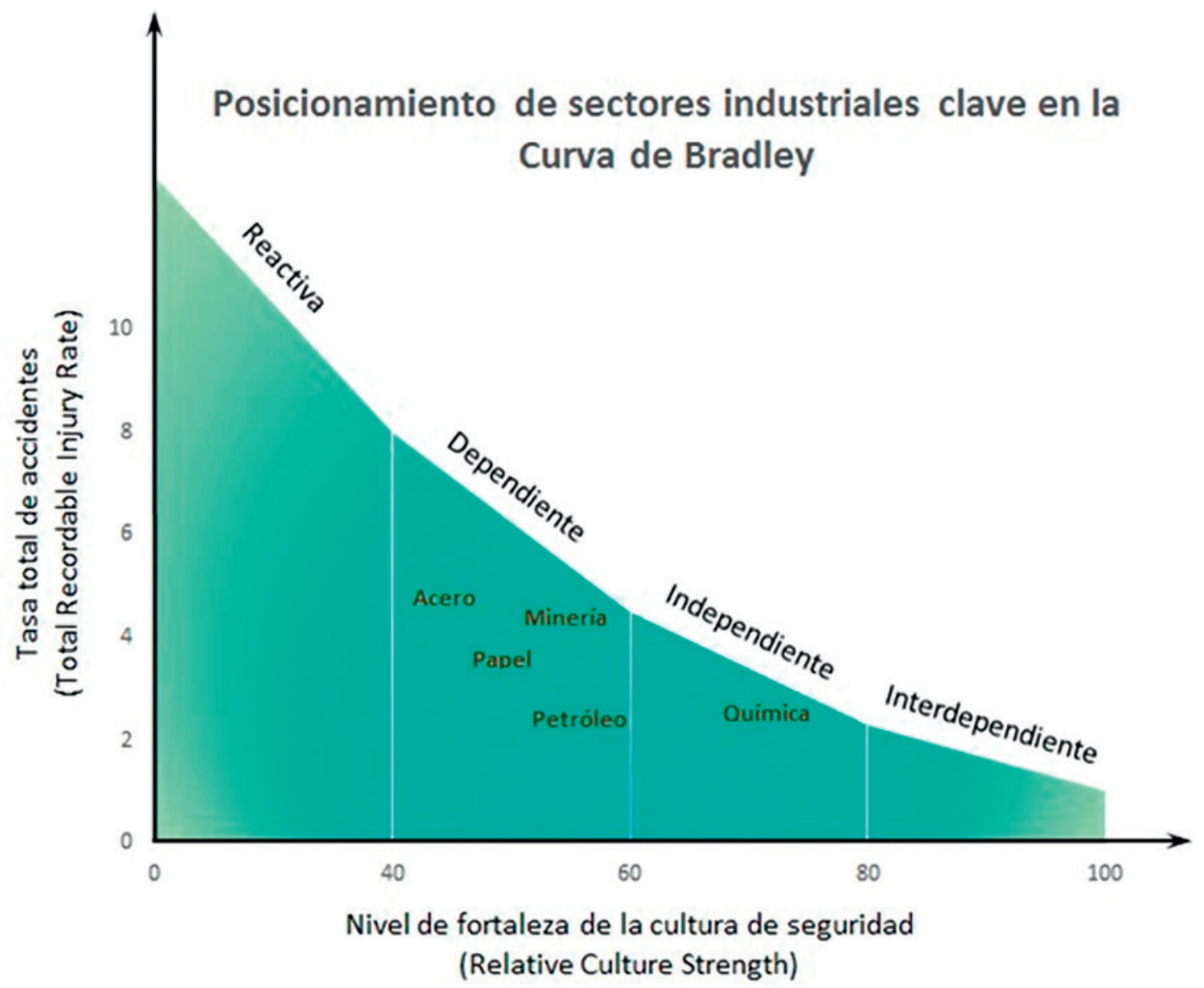

Figura 2. Posicionamiento de sectores industriales clave en la Curva de Bradley Fuente: Oliva (2016) 


\section{RESULTADOS}

En la Tabla 1 y Figura 3, se muestra los valores distributivos de las frecuencias porcentuales de la variable cultura de seguridad minera. La agrupación de indicadores reportados como 'Escasa cultura de seguridad minera' agrupa a los valores de las repuestas dadas en los ítems 'Totalmente en desacuerdo' y 'En desacuerdo' correspondientes a la cultura de seguridad minera. En el resultado 'Mediana cultura de seguridad minera' se muestran los indicadores de las marcas correspondientes a ' $\mathrm{Ni}$ de acuerdo ni en desacuerdo' de las afirmaciones referentes a la cultura de la seguridad minera. El 8.82\% de los estudiantes de Ingeniería de Minas indicaron que tienen mediana cultura de seguridad minera. En cuanto a los indicadores aglutinados como 'Buena cultura de seguridad minera son las respuestas marcadas en 'De acuerdo', 'Totalmente de acuerdo' contenidas en la cultura de seguridad minera, resultando que y el $57.35 \%$ de los estudiantes de Ingeniería de Minas están en buena situación en cuanto a la cultura de la seguridad minera.
De los resultados estadísticos, en la Tabla 2 se tiene que existe una relación $r=0,659$ entre las variables la seguridad corporativa y la cultura de la seguridad minera; valor que indica una correlación positiva considerable. Se encontró una significatividad bilateral de 0,0015704109 , valor menor a 0,01 con lo cual se determina rechazar la hipótesis nula y aceptar la hipótesis alternativa; concluyéndose la dación de una relación directa y significativa entre la seguridad corporativa y la cultura de la seguridad minera en en el ambito del estudio.

En la Tabla 3 la significatividad bilateral obtenida es 0,001892506 , valor menor a 0,01 con lo cual se acepta la hipótesis alternativa. Otro resultado obtenido es que el coeficiente de correlación es $r=0,7503(75,03 \%)$, indicando una relación fuerte entre la Fase reactiva de la seguridad corporativa y la Cultura de la seguridad minera. Valor indicativo de una relación directa entre las variables correlacionadas y, por ende se determina que entre la Fase

Tabla 1. Valores de las frecuencias porcentuales de la variable Cultura de seguridad minera

\begin{tabular}{lcc}
\hline Alternativa & Frecuencia & Porcentaje \\
\hline Escasa cultura de seguridad minera & 23 & 33.82 \\
Mediana cultura de seguridad minera & 6 & 8.82 \\
Buena cultura de seguridad minera & 39 & 57.35 \\
Total & 68 & 100.00 \\
\hline
\end{tabular}

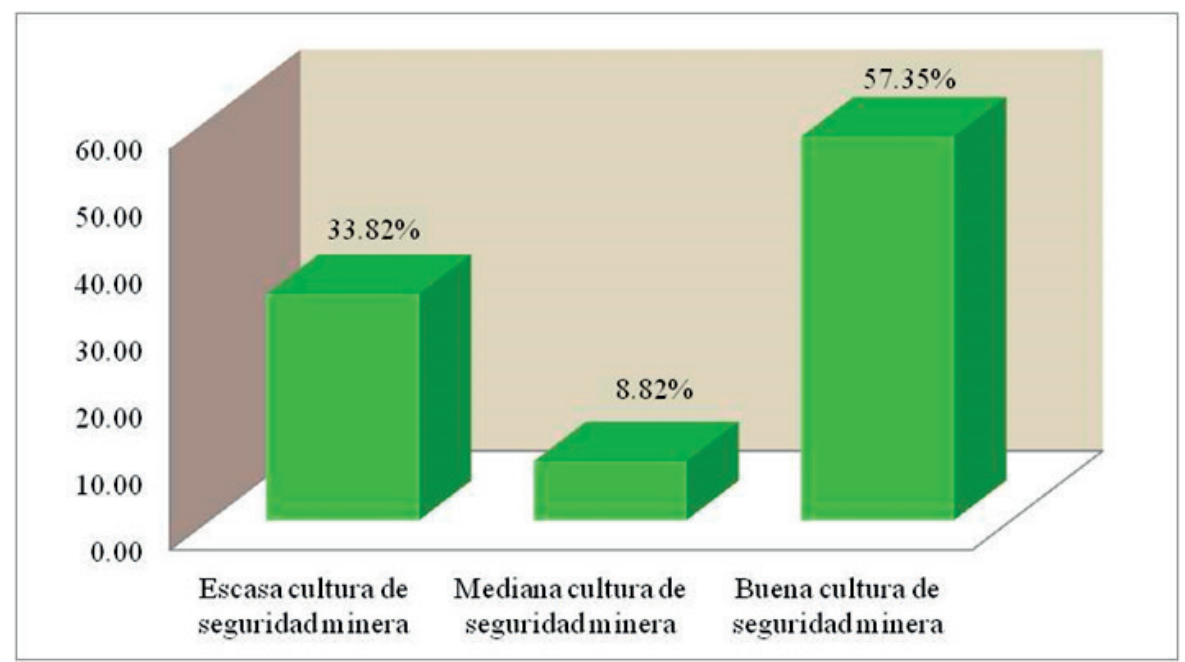

Figura 3. Distribución de frecuencias y porcentajes de la variable cultura de seguridad minera

Tabla 2. Coeficiente de correlación de Spearman de las variables: Seguridad corporativa y Cultura de la seguridad minera

\begin{tabular}{|c|c|c|c|c|}
\hline & & & Seguridad corporativa & Cultura de la seguridad minera \\
\hline \multirow{6}{*}{ Rho de Spearman } & \multirow{3}{*}{ Seguridad corporativa } & Coeficiente de correlación & 1,000 & $r=0,659^{* *}$ \\
\hline & & Sig. (bilateral) & & Sig. $=, 0015704109$ \\
\hline & & $\mathrm{N}$ & 68 & 68 \\
\hline & \multirow{3}{*}{ Cultura de la seguridad minera } & Coeficiente de correlación & $r=0,659^{* *}$ & 1,000 \\
\hline & & Sig. (bilateral) & Sig. $=, 0015704109$ & \\
\hline & & $\mathrm{N}$ & 68 & 68 \\
\hline
\end{tabular}

**. La correlación es significativa al nivel 0,01 (bilateral) 
reactiva de la seguridad en la empresa y la Cultura de la seguridad minera hay una relación positiva y significativa.

En la Tabla 4 la significatividad bilateral entre la Fase dependiente y la Cultura de seguridad minera es de 0,000094573 , resultado menor a 0,01, indicando una relación significativa, y por ende se acepta la hipótesis alternativa. El coeficiente de correlación existente entre la Fase dependiente de la seguridad corporativa y la Cultura de la seguridad minera es $r=0,6501(65,01 \%)$, señalando una relación directa proporcionalmente y con una correlación lineal media; determinándose que entre la Fase dependiente de la seguridad corporativa y la Cultura de la seguridad minera hay una relación positiva y significativa.

En la Tabla 5 la Fase independiente y la Cultura de la seguridad minera tienen una significatividad bilateral de 0,000167024 , resultado menor a 0,01 indicativo para rechazar la hipótesis nula por ende aceptar la hipótesis alternativa. En cuanto al coeficiente de correlación entre la
Fase independiente de la seguridad corporativa y la Cultura de la seguridad minera se obtuvo un $r=0,609(60,9 \%)$; con este valor, la relación es directamente proporcional y una correlación media. Con estos resultados entre la fase independiente de la seguridad corporativa y la cultura de la seguridad minera se tiene una relación positiva y significativa.

Entre la Fase interdependiente y la Cultura de la seguridad minera se obtuvo una significatividad bilateral de 0,000170683 , valor a 0,01 , indicando una la relación significativa y que permite aceptar la hipótesis alternativa. En cuanto al coeficiente de correlación entre la Fase interdependiente de la seguridad corporativa y la Cultura de la seguridad minera el $r=0,6004(60,04 \%)$; con este nivel de correlación se tiene que la relación entre estas variables es directamente proporcional y una correlación lineal media. Con estos resultados se tiene que entre la Fase interdependiente de la seguridad corporativa y la Cultura de la seguridad minera hay una relación positiva y significativa (Ver Tabla 6).

Tabla 3. Correlación entre la Fase reactiva y la Cultura de la seguridad minera

\begin{tabular}{lllll}
\hline & & Fase reactiva & \multicolumn{1}{c}{ Cultura de la seguridad minera } \\
\hline & & Coeficiente de correlación & 1,000 & $r=0,7503^{* *}$ \\
Fho de Spearman & Sig. (bilateral) & & Sig. $=, 001892506$ \\
& & $\mathrm{~N}$ & 68 & 68 \\
& & Coeficiente de correlación & $r=0,7503^{* *}$ & 1,000 \\
& Cultura de la seguridad minera & Sig. (bilateral) & Sig. $=, 001892506$ & \\
& & $\mathrm{~N}$ & 68 & 68 \\
\hline
\end{tabular}

**. La correlación es significativa al nivel 0,01 (bilateral).

Tabla 4. Correlación entre las variables: Fase dependiente y la cultura de la seguridad mineraç

\begin{tabular}{|c|c|c|c|c|}
\hline & & & Fase dependiente & Cultura de la seguridad minera \\
\hline \multirow{6}{*}{ Rho de Spearman } & \multirow{3}{*}{ Fase dependiente } & Coeficiente de correlación & 1,000 & $r=0,6501^{* *}$ \\
\hline & & Sig. (bilateral) & & Sig. $=, 000094573$ \\
\hline & & N & 68 & 68 \\
\hline & \multirow{3}{*}{ Cultura de la seguridad minera } & Coeficiente de correlación & $r=0,6501^{* *}$ & 1,000 \\
\hline & & Sig. (bilateral) & Sig. $=, 000094573$ & \\
\hline & & $\mathrm{N}$ & 68 & 68 \\
\hline
\end{tabular}

**. La correlación es significativa al nivel 0,01 (bilateral).

Tabla 5. Correlación entre las variables: Fase independiente y la cultura de la seguridad minera

\begin{tabular}{lllll}
\hline & & \multicolumn{1}{c}{ Fase independiente } & \multicolumn{1}{c}{ Cultura de la seguridad minera } \\
\hline & \multirow{4}{*}{ Fase independiente } & Coeficiente de correlación & 1,000 & $r=0,609^{* *}$ \\
Rho de Spearman & Sig. (bilateral) & & Sig. $=, 000167024$ \\
& & $\mathrm{~N}$ & 68 & 68 \\
& & Coeficiente de correlación & $r=0,609^{* *}$ & 1,000 \\
& Cultura de la seguridad minera & Sig. (bilateral) & Sig. $=, 000167024$ & \\
& & $\mathrm{~N}$ & 68 & 68 \\
\hline
\end{tabular}

**. La correlación es significativa al nivel 0,01 (bilateral). 
Tabla 6. Correlación entre las variables: Fase interdependiente y la cultura de la seguridad minera

\begin{tabular}{lllll}
\hline & & \multicolumn{1}{c}{ Fase Interdependiente } & \multicolumn{1}{c}{ Cultura de la seguridad minera } \\
\hline & \multirow{4}{*}{ Fase Interdependiente } & Coeficiente de correlación & 1,000 & $r=0,6004^{* *}$ \\
Rho de Spearman & Sig. (bilateral) & & Sig. $=, 000170683$ \\
& & $\mathrm{~N}$ & 68 & 68 \\
& & Coeficiente de correlación & $r=0,6004^{* *}$ & 1,000 \\
& \multirow{2}{*}{ Cultura de la seguridad minera } & Sig. (bilateral) & Sig. $=, 000170683$ & \\
& & $\mathrm{~N}$ & 68 & 68 \\
\hline
\end{tabular}

**. La correlación es significativa al nivel 0,01 (bilateral).

\section{RESULTADOS}

En el presente estudio se encontró que entre la seguridad corporativa y la cultura de la seguridad existe relación significativa, de manera que los estudiantes de ingeniería de minas observan que en las empresas mineras existe el compromiso corporativo de interiorizar la seguridad en la organización; lo cual es apuntalado por Falconi \& Romero (2020), quienes precisan que un sistema de seguridad y salud ocupacional debe ser parte de la gestión de seguridad de una empresa e incluso en una MIPYME, como parte de una cultura empresarial responsable. Es un resultado que también es cierta manera es corroborada por Huapaya \& Calderón (2017), quien precisa que el método de gestión MHL, mediante una cultura de prevención, incide significativamente en la reducción de accidentes laborales, con ello se tiene que la cultura de la seguridad es un elemento para afrontar los actos inseguros que devienen en accidentes de trabajo. Por otro lado, se tiene que Hellenbroich et al. (2015) encontraron que en las distintas áreas de una empresa de hidrocarburos los procedimientos de seguridad no están interiorizados ni se practican cabalmente, rebelando que falta interiorizar la cultura de la seguridad en la empresa y que existe el compromiso de la alta dirección para generar la cultura de la seguridad, un resultado concordante con Rodríguez, P.; Calderón, M.; Berrocal, L. \& Medina (2018), quienes encontraron que la empresa donde realizó el estudio falta trabajar sobre el factor actitudinal ante la seguridad, pues se debe trabajar en la motivación conductual a fin de fortalecer en el trabajador una actitud de proactiva a situaciones de riesgo, que es lo que se busca con una cultura de seguridad.

\section{CONCLUSIONES}

En los estudiantes de Ingeniería de Minas de la Universidad Nacional de Moquegua existe relación positiva y significativa entre la seguridad corporativa y la cultura de la seguridad minera, manifestada en que el $57.35 \%$ muestra una buena cultura de seguridad minera, y que dada su etapa formativa están en la fase reactiva de la cultura de la seguridad, lo cual debe ir mejorarndo con la culminación de sus estudios y en la medidad que desarrollen su experiencia laboral.

\section{REFERENCIAS}

Cajia Lucio, T., \& Silva, W. (2019). Influencia de la metodologia seguridad basada en el comportamiento en la prevención y reducción del número de accidentes en CAME Contratistas y Servicios Generales S.A. - Proyecto Antamina - Periodo 2014. 22, 93-98. https://revistasinvestigacion.unmsm.edu. pe/index.php/iigeo/article/view/16693

Cárdenas, J., Arcos, A., \& Echevarría, E. (2017). Seguridad y salud en la pequeña minería colombiana: estudios de caso en oro y carbón. In Alianza por la Minería Responsable. CAlianza por la Minería Responsable, proyecto Somos Tesoro, financiado por el Departamento de Trabajo de los Estados Unidos. http://www.responsiblemines.org/ wp-content/uploads/2017/06/Somos-Tesoro-Seguridad-ySalud.compressed.pdf

Ceja Rodriguez, J. A. (2019). Construyendo la seguridad corporativa. Simposio de seguridad. ANTAD. https://antad. net/simposio-seguridad-2019/

Committee of Experts on Management of Safety and Quality in Health Care. (2005). Expert Group on Safe Medication Practices - Glossary of terms related to patient and medication safety. In Council of Europe. https://www.who. int/patientsafety/highlights/COE patient and medication safety_gl.pdf

Diario El Peruano. (2016). Decreto Supremo N 024-2016-EM. Ministerio de Energía y Minas. https://busquedas.elperuano. $\mathrm{pe} /$ normaslegales/aprueban-reglamento-de-seguridad-ysalud-ocupacional-en-mine-decreto-supremo-n-024-2016em-1409579-1/

Falconi Agapito, F., \& Romero Baylon, A. (2020). Las micro, pequeña y mediana empresa y sus adaptación a la normativa de seguridad y la salud en el trabajo. Revista Del Instituto de Investigación de La Facultad de Ingeniería Geológica, Minera, Metalúrgica y Geográfica, 23(45), 17-28. https:// doi.org/10.15381/iigeo.v23i45.18058

Hellenbroich Landra, C., Reyes Oliva, P., \& Torres García, F. (2015). Diagnóstico y propuesta de mejora de la cultura de seguridad en una empresa de hidrocarburos del Perú, en los lotes en tierra y área administrativa. Repositorio de La Universidad Del Pacifico - UP. https://repositorio.up.edu. pe/handle/11354/1816

Hernándes Sampieri, R., \& Mendoza Torres, C. P. (2018). Metodología de la investigación - Las rutas cuantitativa, cualitativa y mixta. In Mac graw hill education. http:// virtual.cuautitlan.unam.mx/rudics/?p=2612

Herrera, I., \& Parra, A. (2018). La actividad minera y el lugar que ocupa en la economia mexicana del siglo XIX. Caravelle, 111, 11-24. https://doi.org/10.4000/caravelle.3575

Huapaya, O., \& Calderón, M. (2017). Influencia del método MHL para reducir los accidentes laborales mediante una cultura 
de prevención, actos y condiciones seguras en las plantas de producción de CuSO4.5H2O. Revista Del Instituto de Investigación de La Facultad de Ingeniería Geológica, Minera, Metalurgica y Geográfica, 19(38), 85-90. https:// revistasinvestigacion.unmsm.edu.pe/index.php/iigeo/ article/view/13572

Iberdrola. (2013). Politica de Seguridad Corporativa. 2. https://www.mendeley.com/catalogue/c590fedc-24343ddb-8bc9-975406d74de9/?utm source=desktop\&utm medium $=1.19 .8 \& u$ tm_campaign $=$ open_catalog \&u serDocumentId=\% 7B257bdad4-5b18-3724-b45b$3 \mathrm{f} 7 \mathrm{c} 3 \mathrm{ab} 545 \mathrm{~b} 4 \% 7 \mathrm{D}$

ICSI. (2017). Lo esencial de la cultura de Seguridad. Institute Pour Une Culture de Segurite Industrialle, 1-15. https:// www.icsi-eu.org/sites/default/files/2020-07/Icsi_esencial_ ES_cultura-seguridad_2017.pdf

Iglesias de Ussel, J., Trinidad Requena, A., \& Soriano Miras, R. M. (2016). La sociedad desde la Sociología : una introducción a la Sociología General (Tecnos (ed.)). Tecnos. https:// www.tecnos.es/ficha.php?id=5730238\&id_clase $=219190$

IPE. (2017). El valor agregado de la minería. In Instituto de Estudios Energético Minero. https://www.mendeley.com/ catalogue/e61ac584-fa5f-3973-b1dd-502e97f253de/?utm source=desktop\&utm_medium $=1.19 .8 \& u$ tm campaign $=$ open_catalog\&userDocumentId $=\% 7 \mathrm{~B} 2$ adadd 3f-0198-3835-87fa-bebbc088d137\%7D

Mejia, C. R., Cárdenas, M. M., \& Gomero-Cuadra, R. (2015). Notificación de accidentes y enfermedades laborales al Ministerio de Trabajo. Perú 2010-2014. Revista Peruana de Medicina Experimental y Salud Pública, 32(3), 526. https:// doi.org/10.17843/rpmesp.2015.323.1689

Muñoz,J.(2016).SeguridadOrganizacional-SeguridadIntegrada. Seguritecnia. https://www.seguritecnia.es/tecnologias-yservicios/seguridad-organizacional_20160910.html
Namakforoosh, M. N. (2015). Metodología de la investigación. Librería LIMUSA , $2^{\circ}$ Ed. https://libreria-limusa.com/ producto/metodologia-de-la-investigacion-2a-ed/

Oliva Serrano, A. (2016). ¿Qué nos enseña la curva de Bradley? Preven Control. https://prevencontrol.com/prevenblog/nosensena-la-curva-bradley/

Pérez López, M. H. (2015). ¿ Cómo Generar Cultura Preventiva ? Asociación de Especialistas En Prevencion y Salud Laboral, 1-19. https://www.diba.cat/documents/467843/44174644/ ComoGeneralCulturaPreventiva_28-4-2015.pdf/51a36b0133b1-4ce1-b8d9-d31f2e899a35

Rodríguez, P.; Calderón, M.; Berrocal, L. \& Medina, A. (2018). OCAS - Generando una Cultura de Seguridad. Revista Del Instituto de Investigación de La Facultad de Ingeniería Geológica, Minera, Metalurgica y Geográfica, 20(40), 4247. https://revistasinvestigacion.unmsm.edu.pe/index.php/ iigeo/article/view/14388

Seguel Conejeros, K., Navarrete Espinoza, E., \& Bahamondes Valenzuela, G. (2017). Explicación de la Accidentabilidad Laboral Basada en Factores de Riesgo Psicosocial y Rasgos de Personalidad en el Transporte Forestal. Ciencia \& Trabajo, 19(60), 157-165. https://doi.org/10.4067/s071824492017000300157

Valdez, Y. (2016). Breve resumen del impacto de la minería en el Perú. May, 0-9. https://www.researchgate.net/ publication/304824894_Breve_resumen_del_impacto_de_ la_mineria_en_el_Peru

Vázquez, A. (2018). Los pilares de la seguridad corporativa: presente y futuro. RECERCAT (Dipòsit de La Recerca de Catalunya), 202. http://www.recercat.cat/ handle/2072/338148

Vítolo, F. (2016). Cultura de Seguridad. Noble Compañia de Seguros, 1-13. http://www.noble-arp.com/pdf/cultura_de seguridad.pdf 